\title{
Skills of novices early trained or traditionaly trained versus experienced drivers confronted to simulated urban accidents' scenarios
}

\author{
Catherine Berthelon ${ }^{\mathrm{a}, *}$, Loïc Damm ${ }^{\mathrm{b}}$ \\ a IFSTTAR, MA, F-13300 Salon de Provence, France \\ ${ }^{b}$ University of Exeter, St Luke's Campus, Heavitree Road, EX1 2LU, Exeter, United Kingdom
}

\begin{abstract}
In order to prevent the over-representation of young drivers in car crashes, France instated an early driver training from the age of 16, but the positive effects of this opportunity have not yet been proven. Three groups of male drivers (12 subjects each) were confronted with some prototypical accident scenarios introduced in a simulated urban circuit. The first and second groups were composed of young drivers having less than one month of driving licence; twelve have had a traditional learning course, and twelve had followed, in addition to the initial course, an early driver training under the supervision of an adult. The third group was composed of experienced drivers. Strategies of the three groups were analyzed through their response time, speed and maneuvers. No difference appeared across groups regarding obstacle detection. But traditionallytrained drivers' position control was more conservative than the two others groups, which were more likely to involve efficient evasive action. The exposure gained during early training could thus increase the development of visuo-motor coordination and involve better skills in case of difficult situations. Others accidents' scenarios could be used to confront young drivers with difficult situations not commonly encountered in natural driving.
\end{abstract}

Keywords: driving experience, simulator, scenario of accident, early training

\section{Introduction}

The overall representation of young drivers in car crashes is a well-established fact in many countries $[1,2]$. However, the influence of both experience and age contributes to confusion in identifying the primary factors responsible for this higher accident rate.

The difficulty of the analysis comes from the fact that experience, represented by the number of years the person has had a driving license, and age simultaneously affect the development of driving skills: isolating these effects presupposes comparing homogeneous groups, which requires rigorous efforts to avoid methodological bias. The fulfilment of this prerequisite is actually tricky, considering the systematic licensing of most of drivers at the same age. A review of recent studies focused on young drivers, [3] tried to evaluate the respective effects of these two parameters. One year of additional experience appears to bring stronger positive effects than being one year older at the age of licensure $[1,4]$.

The question of the minimum experience necessary to record advantageous effects seems to be crucial. Since 1988, young French people can start early driver training (in French, Apprentissage Anticipe de la Conduite) at the age of 16 . The initial training is quite similar to traditional training, but the learner acquires additional experience consisting in driving with an adult for at least $3000 \mathrm{kms}$. The evaluation of this training method comes up against the amount of practice and the diversity of driving situations the learner encounters. The objective of the present research was to compare the behavior of novice drivers with early driver training to novice drivers with traditional driver training in order to evaluate the capacities of these two types of learning to to develop similar driving skills than the ones of experienced drivers.

*Corresponding author: catherine.berthelon@ifsttar.fr 


\section{Method}

Three groups of male drivers (12 subjects each) were confronted with some prototypical accident scenarios, i.e. a set of prototypical situations known to generate accidents, introduced in a simulated urban circuit. The first and second groups were composed of young drivers having less than one month of driving licence; twelve of them have had a traditional learning course, and twelve had followed, in addition to the initial course, an early driver training under the supervision of an adult. The third group was composed of experienced drivers (less than 28 years old having held a driving license for more than 3 years). Strategies of the three groups were analyzed through their response time, speed and lateral position at the moment origin of the scenarios.

The simulator consisted of a fixed-base, one quarter vehicle and 3 video projectors (angular opening of $150^{\circ}$ horizontally and $40^{\circ}$ vertically). Drivers controlled the vehicle using the steering wheel and the accelerator.

The subjects were asked to drive in an urban environment at a speed of $50 \mathrm{~km} . \mathrm{h}-1$. Before the experiment, they drove a first circuit which did not include any accident scenarios. Five accident scenarios were introduced randomly in the experimental session, which included two rounds lasting approximately 8 minutes each. No information was given regarding the events to come.

Overtaking scenario: a vehicle overtakes the driver's vehicle and merges back into his lane (see Figure 1).

Pedestrian scenario: a pedestrian, initially hidden by a bus parked on the right-hand side of the carriageway, crosses.

Station scenario: a vehicle driving in the opposite lane suddenly turns left in front of the participant.

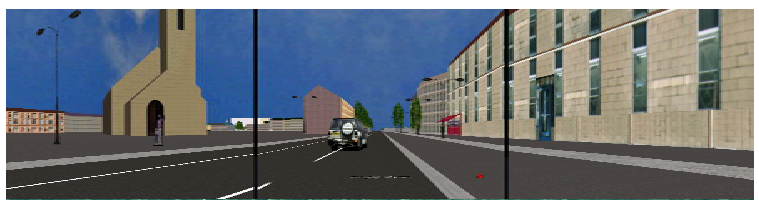

Fig. 1. Overtaking scenario.

Intersection scenario: a vehicle arrives at very low speed from the left at an intersection.

Parking scenario: a vehicle pulls out from its parking place.

\section{Result}

Results only showed 8 accidents whose 4 due to the traditional training group of participants and 2 due to each of the two others groups of participants.

No difference appeared across groups regarding obstacle detection as revealed by the response times (trad., $\mathrm{m}=1.36$ and $\mathrm{SD}=0.23$; early, $\mathrm{m}=1.27$ and $\mathrm{SD}=0.22$; exp., $\mathrm{m}=1.18$ and $\mathrm{SD}=0.2$ ).

Lateral position at the moment origin of the scenarios significantly varied with the scenarios $(F(4,132)=166.32, p<.001$; Figure 2$)$ and there was an interaction between group and scenarios $(\mathrm{F}(8,132)=4.82, \mathrm{p}<.001)$. Overtaking and station scenarios produced significant different lateral position between traditional and early training group of drivers $(\mathrm{p}<.05)$, pedestrian and parking scenarios produced significant more to the right lateral position for traditional than for early training groups and for traditional training than for experienced group $(\mathrm{p}<.01)$.

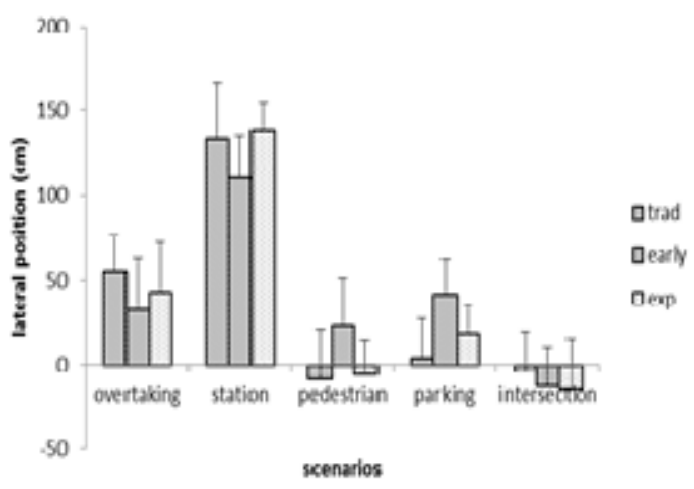

Fig. 2. Lateral position at the moment origin of the scenarios as a function of group and scenarios.

Mean speed at the moment origin of the scenarios did not vary significantly between groups $(\mathrm{F}(2,33)=2.63$, NS) and there was no interaction $(F(8,132)=.85$, ns; Figure 3). The mean speeds measured at the origin of the scenarios (T0) corresponded only to the prescribed speed for the overtaking scenario $(\mathrm{M}=50.13 \mathrm{~km} / \mathrm{h}), \mathrm{t}(35)=0.23, \mathrm{p}<.82$. Speeds were below the instructed value for all other scenarios: hidden pedestrian $(\mathrm{M}=45.9 \mathrm{~km} / \mathrm{h}), \mathrm{t}(35)=$ $-4.04, \mathrm{p}<.01$; opposite vehicle crossing $(\mathrm{M}=43.2$ $\mathrm{km} / \mathrm{h}), \mathrm{t}(35)=-6.71, \mathrm{p}<.01 ;$ left $\operatorname{crossroad}(\mathrm{M}=41.3$ $\mathrm{km} / \mathrm{h}), \mathrm{t}(35)=-7.59, \mathrm{p}<.01$; parked vehicle $(\mathrm{M}=$ $37.4 \mathrm{~km} / \mathrm{h}), \mathrm{t}(35)=-9.59, \mathrm{p}<.01$. 


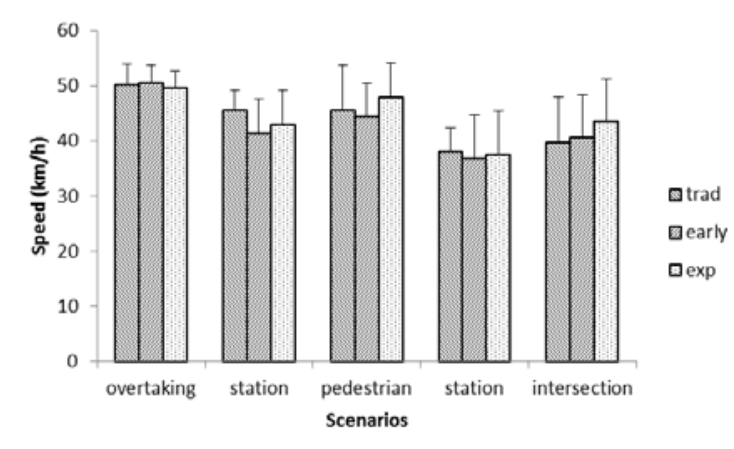

Fig.3. Speed as a function of group and scenarios.

\section{Conclusion}

The speed did not differ across groups at the beginning of scenarios: all groups reached the onset of each scenario with comparable speeds and experienced the same initial conditions to build their anticipatory behaviour.

Whatever the group, reaction times of the participants were similar. Generally, lack of experience appears to be a disadvantage in foreseeing risky situations and this result is not in agreement with past studies [5]. In the present study, young drivers appeared to be able to detect the onset of a risky scenario as early as more experienced drivers. More stringent conditions like distracting events or ambiguous situations, could possibly contribute to reveal some differences across groups [6].

Traditionally-trained drivers' position control was more conservative than the two others groups, as showed by their straighter trajectory compared to other groups during overtaking and pedestrian scenarios. Significant difference emerged when the time constraint of the scenario was high and when the upcoming event was unpredictable. Traditionallylearned drivers also differentiated themselves from other groups by their overall positioning in the lane. In 2 scenarios (parked vehicle and opposite vehicle crossing), their position was clearly more toward the right of the lane. These results could be linked to the novice drivers' strategy for scanning the environment. Novice drivers tend to monitor both the foreground and the right of the vehicle for direct control of their lateral position [7].

to the right lateral position in two of our scenarios. This difference was significant when the time constraint of the scenario was high and when the future critical event was unpredictable as in pedestrian and parking scenarios.

The exposure gained during early training could thus increase the development of visuo-motor coordination and involve better skills in case of difficult situations [8]. The fact that the simulator used in this research did not have a gearbox may however have masked inter-group differences. Others scenarios of accidents need to be included in the driving simulators and could be used to confront young drivers with ranges of difficult situations not commonly encountered in natural driving. Using this approach before licensing, the use of simulators could supplement young drivers' experience.

\section{References}

[1] D.R. Mayhew, H.M. Simpson and A. Pak, Changes in collision rates among novice drivers during the first months of driving, Accident Analysis \& Prevention 35 (2003), 683 - 691.

[2] A.F. Williams and V.I. Shabanova, Responsibility of drivers, by age and gender, for motor-vehicle crash deaths, Journal of Safety Research 34 (2003), 527-531.

[3] A.T. McCartt et al., Effects of age and experience on young driver crashes: review of recent literature, Traffic Injury Prevention 10 (2009), $209-219$.

[4] P.F. Waller et al., Changes in young adult offense and crash patterns over time, Accident Analysis \& Prevention 33 (2001), $117-128$.

[5] A. Borowsky, T Oron-Gilad and Y, Parmet, Age and skill differences in classifying hazardous traffic scenes, Transportation Research Part F: Traffic Psychology and Behaviour 12 (2009), $277-287$.

[6] D. E. Crundall and G. Underwood, (1998). Effects of experience and processing demands on visual information acquisition in drivers, Ergonomics 41(4), 448 - 458

[7] A. Smiley, L., Reid, and M. Fraser, Changes in Driver Steering Control With Learning, Human Factors: The Journal of the Human Factors and Ergonomics Society, 22(15), (1980), 401-415.

[8] L. Damm, C. Nachtergaële, M. Meskali and C. Berthelon, The evaluation of traditional and early driving learning with simulated accident scenarios. Human Factors, 53 (2011), 323 - 337. 